\title{
Map-Based Cloning, Phylogenetic, and Microsynteny Analyses of ZmMs20 Gene Regulating Male Fertility in Maize
}

\author{
Yanbo Wang ${ }^{1,+}{ }^{\text {, Dongcheng Liu }}{ }^{1,2,+}$, Youhui Tian ${ }^{1,+}$, Suowei $\mathrm{Wu}^{1,2}$, Xueli An ${ }^{1,2}$, \\ Zhenying Dong ${ }^{1,2}$, Simiao Zhang ${ }^{1}$, Jianxi Bao ${ }^{1}$, Ziwen $\mathrm{Li}^{1,2}$, Jinping $\mathrm{Li}^{2}$ \\ and Xiangyuan Wan $1,2, * \mathbb{D}$ \\ 1 Biology and Agriculture Research Center, University of Science and Technology Beijing, Beijing 100024, \\ China; b20180389@xs.ustb.edu.cn (Y.W.); dongchengliu@ustb.edu.cn (D.L.); tianyouhui@ustb.edu.cn (Y.T.); \\ suoweiwu@ustb.edu.cn (S.W.); xuelian@ustb.edu.cn (X.A.); zydong@ustb.edu.cn (Z.D.); \\ zhangsimiao11@126.com (S.Z.); bjx1232003@126.com (J.B.); ziwen.li@163.com (Z.L.) \\ 2 Beijing Engineering Laboratory of Main Crop Bio-Tech Breeding, Beijing International Science and \\ Technology Cooperation Base of Bio-Tech Breeding, Beijing Solidwill Sci-Tech Co. Ltd., \\ Beijing 100192, China; lijinping@sjlhtech.com \\ * Correspondence: wanxiangyuan@ustb.edu.cn or wanxiangyuan@sjlhtech.com; Tel.: +86-10-6299-5866 \\ + These authors contributed equally to this work.
}

Received: 26 January 2019; Accepted: 19 March 2019; Published: 20 March 2019

\begin{abstract}
Genic male sterility (GMS) mutant is a useful germplasm resource for both theory research and production practice. The identification and characterization of GMS genes, and assessment of male-sterility stability of GMS mutant under different genetic backgrounds in Zea may (maize) have (1) deepened our understanding of the molecular mechanisms controlling anther and pollen development, and (2) enabled the development and efficient use of many biotechnology-based male-sterility (BMS) systems for hybrid breeding. Here, we reported a complete GMS mutant (ms20), which displays abnormal anther cuticle and pollen development. Its fertility restorer gene ZmMs20 was found to be a new allele of IPE1 encoding a glucose methanol choline (GMC) oxidoreductase involved in lipid metabolism in anther. Phylogenetic and microsynteny analyses showed that ZmMs20 was conserved among gramineous species, which provide clues for creating GMS materials in other crops. Additionally, among the 17 maize cloned GMS genes, ZmMs20 was found to be similar to the expression patterns of Ms7, Ms26, Ms6021, APV1, and IG1 genes, which will give some clues for deciphering their functional relationships in regulating male fertility. Finally, two functional markers of $\mathrm{ZmMs} 20 / \mathrm{ms} 20$ were developed and tested for creating maize $m s 20$ male-sterility lines in 353 genetic backgrounds, and then an artificial maintainer line of $m s 20$ GMS mutation was created by using ZmMs 20 gene, $m s 20$ mutant, and BMS system. This work will promote our understanding of functional mechanisms of male fertility and facilitate molecular breeding of $m s 20$ male-sterility lines for hybrid seed production in maize.
\end{abstract}

Keywords: genic male sterility (GMS); anther development; glucose methanol choline (GMC) oxidoreductase; hybrid breeding; Zea may

\section{Introduction}

Male sterility is a common phenomenon in higher plants and widely used in crop hybrid seed production. According to the inheritance characteristic, it can be divided into cytoplasmic male sterility (CMS) and genic male sterility (GMS). Generally, CMS is caused by a toxic protein in the cytoplasm, and the fertility can be rescued by restorer genes in the nucleus. GMS usually results from the loss-function of genic genes, mainly participating in anther and pollen development. Anther 
locules are composed of center-localized meiocyte cells, which are surrounded by four somatic cell layers. From stamen primordia initiation to mature pollen formation, thousands of genes likely take part in this complicated process. So far, hundreds of GMS mutants have been found, while only 17 GMS genes have been cloned in maize. Among them, nine genes were reported to participate in the ontogeny of anther cuticle and pollen exine [1].

The anther cuticle locates outside of the epidermis. It acts as a barrier to transpiration, pathogens, and other stresses [2]. The anther cuticle contains cutin and wax. Cutin is mainly composed of polyesters derived from hydroxylated and epoxy C16 and C18 fatty acids produces [3], and cuticle wax is a mixture of very-long-chain fatty acid, alkanes, alkenes, and fatty alcohols [4]. To date, some GMS genes participating in anther cuticle development have been isolated in model plants, such as Arabidopsis thaliana, Oryza sativa (rice), and maize. For instance, AtMs2, OsDPW, and ZmMs6021 encode plastid localized reductases catalyzing the reduction of fatty acyl-ACP to corresponding fatty alcohols [5-7]. AtCYP703A2, OsCYP703A3, and ZmAPV1 function as lauric acid hydroxylase [8-10]. AtCYP704B1, OsCYP704B2, and ZmMs26 catalyze the $\omega$-hydroxylation of fatty acid [11-13]. OsGPAT3 and ZmMs33 encode glycerol-3-phosphate acyltransferase with unknown substrates [14-16]. ZmMs30 encodes a Gly-Asp-Ser-Leu (GDSL)-motif lipase with diverged catalytic residues and prefers substrates with short carbon chains [17]. The loss-function of any of the genes mentioned above leads to arrested anther cuticle development. In addition, transcription factors, such as AtMs1, OsPTC1, and ZmMs7, were also reported to regulate anther cuticle development [18-20].

Glucose-methanol-choline (GMC) oxidoreductase family catalyze the oxidation of $\mathrm{CH}-\mathrm{OH}$ to the corresponding aldehyde. The GMC oxidoreductase family includes diverse subclades, such as oxidoreductases, dehydrogenases, lyases, and oxidases [21]. Generally, these members have a wide variety of substrates. For instance, the oxidases can be divided into glucose oxidase, choline oxidase, cholesterol oxidase, cellobiose dehydrogenase, aryl-alcohol oxidase, and pyridoxine 4-oxidase according to the substrates [22]. An algae-specific GMC member can catalyze the decarboxylation of free fatty acids to n-alkanes or n-alkenes in response to blue light [23]. In plants, Arabidopsis ACE/HTH is the first reported GMC gene, and it oxidizes long-chain $\omega$-hydroxy fatty acids produced in the $\omega$-oxidation pathway of cytochrome P450 fatty acid $\omega$-hydroxylases [24]. Its transcripts are detectable in both floral organs and roots; however, ace/hth only shows fusions of floral organs and genetic background-dependent male sterility due to the disruption of the membrane structure [24]. Rice mini1, an ortholog of $a c e / h t h$, is more severely defective, and exhibits a smaller architecture, shortened leaves and sheaths, and withered leaf blade tips $[25,26]$. HTH1 and OsNP1, two other homologous GMC genes specifically expressed in the anther of rice, have been recently reported to participate in anther cuticle development. HTH1 is highly expressed in the anther epidermis and tapetum, and OsNP1 is expressed in the anther tapetum. Both $h t h 1$ and $n p 1$ display a glossy anther surface and aborted pollen grains [27-29]. In maize, IPE1 exhibits similar characteristics to its ortholog OsNP1 [30].

In this study, we found that $m s 20$, a complete male-sterility mutant, displayed an unextruded smaller anther, glossy cuticles, and abnormal pollen grains. ZmMs20 was found to be a new allele of IPE1 and encode a GMC oxidoreductase, which is a small gene family including 8,7, and 6 members in Arabidopsis, rice, and maize, respectively. To explore the potential functions of GMC genes underlying anther development, phylogenetic and expression pattern analyses were performed. The analysis results showed that $\mathrm{ZmMs} 20$ and its orthologs underwent different evolution pathways between monocots and dicots, while they were greatly conserved during the evolution of gramineous species. Additionally, the expression patterns of 17 cloned GMS genes in maize were investigated here. Most of these genes were expressed in anthers with different expression peaks at stages 7, 8, or 9. ZmMs20 was found to be expressed after initiation of meiosis with high expression at stages $8 \mathrm{~b}$ and 9 , similar to the expression patterns of $M s 7, M s 26, M s 6021, A P V 1$, and IG1 genes [7,10,13,20,31]. Finally, two functional markers were developed to create maize $m s 20$ male-sterility lines in different genetic backgrounds by marker-assisted selection (MAS), which could facilitate molecular breeding of ms 20 male-sterility lines for hybrid breeding and seed production in maize. 


\section{Results}

\subsection{Genetic and Phenotypic Analyses of ms20 Mutant}

Maize male-sterility mutant ms20 was originally obtained from the Maize Genetics Operation Stock Center (http:/ / maizecoop.cropsci.uiuc.edu). When the mutant plants were crossed with maize inbred line Chang7-2, all the $\mathrm{F}_{1}$ progenies were fertile. The segregation of fertile to sterile $\mathrm{F}_{2}$ individuals fitted an approximate ratio of 3:1 (Table 1), which uncovered the recessive monofactorial inheritance characteristic of ms20 mutant. Compared with wild type (WT), no obvious difference was observed in $m s 20$ during vegetative growth. However, ms20 anthers could not be exerted out of glumes (Figure 1A1,A2), became smaller and wilted (Figure 1A3,A4), and did not generate mature pollen grains (Figure 1A5,A6). The seed setting of ms20 was normal when pollinated with WT pollens, indicating that the female fertility of $m s 20$ was unaffected.

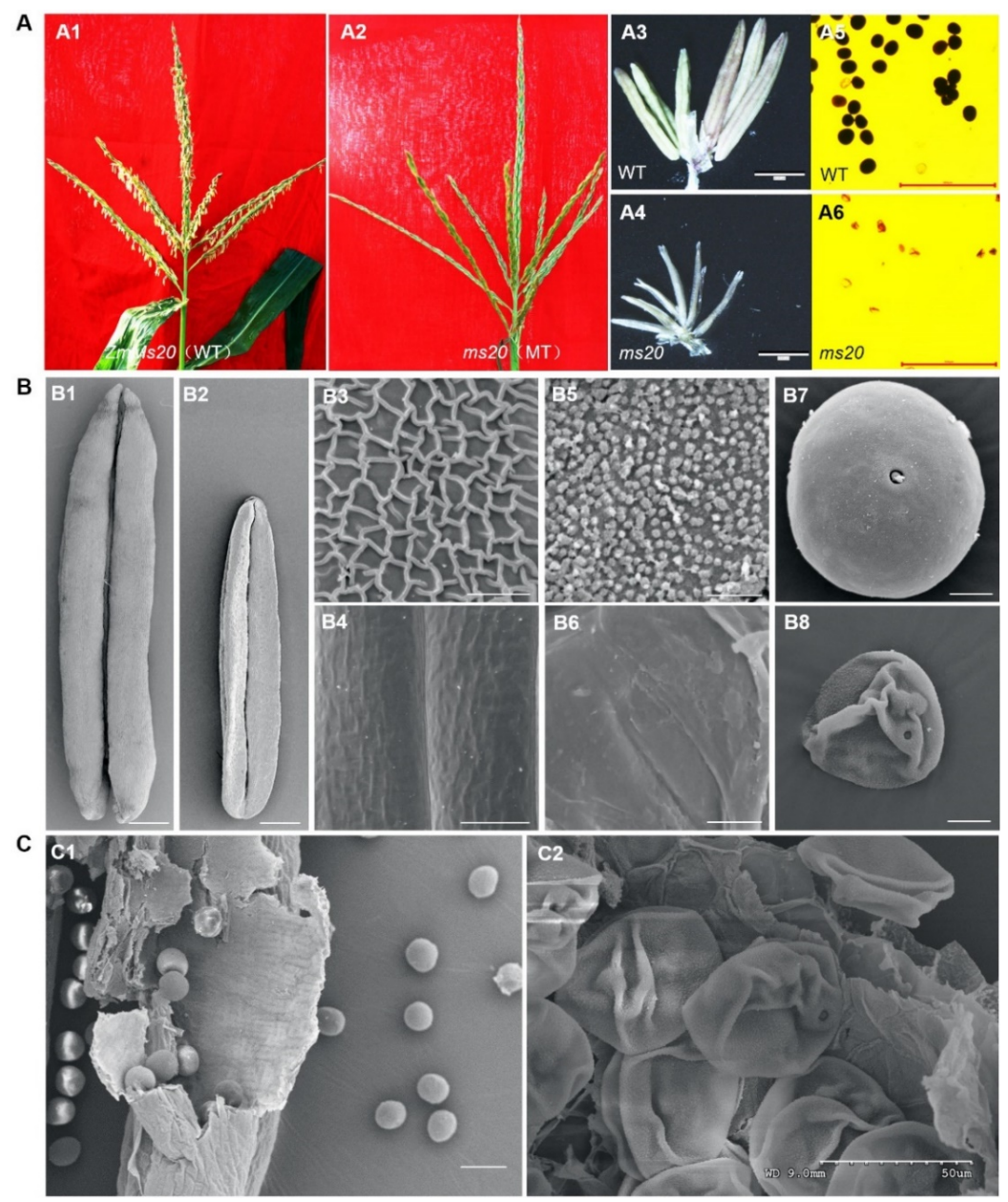

Figure 1. Phenotypic comparison of maize tassels, anthers, and pollen grains between wild type (WT) and ms20 mutant at anthesis stage. (A) Tassels (A1 and A2), spikelet (A3 and A4), and pollen grains stained with $\mathrm{I}_{2}-\mathrm{KI}$ solution (A5 and A6) of WT and $m s 20$. Bars $=2.5 \mathrm{~mm}$ in A3 and A4, $500 \mu \mathrm{m}$ in A5 and A6. (B) SEM analysis of WT and $m s 20$ anthers and pollen grains at anthesis stage. (B1,B2) The $m s 20$ anther (B2) is smaller and wilted than that of WT (B1). (B3,B4) The outer surface of $m s 20$ anther (B4) is glossy compared with that of WT (B3). (B5,B6) Ubisch bodies (B5) were observed on the inner surface of WT anther but none on the $m s 20$ anther $(\mathbf{B 6})$. (B7,B8) The WT pollen grain is round-shape (B7), while $m s 20$ pollen is wizened in the locule (B8). Bars $=500 \mu \mathrm{m}$ in B1 and B2, $5 \mu \mathrm{m}$ in B3 and B4, 2 $\mu \mathrm{m}$ in B5 and B6, and $10 \mu \mathrm{m}$ in B7 and B8. (C) SEM analysis of WT and $m s 20$ pollens at anthesis stage. For $\mathbf{C} 1$ and $\mathbf{C} 2$, WT pollens (C1) are spread out, but $m s 20$ pollens (C2) are stuck on the inner surface of anther locule. Bars $=100 \mu \mathrm{m}$ in $\mathbf{C 1}$, and $50 \mu \mathrm{m}$ in $\mathbf{C} 2$. 
Table 1. The ratio of fertile to sterile plants in the $F_{2}$ population derived from the cross of $m s 20 \times$ Chang7-2.

\begin{tabular}{cccccccc}
\hline $\begin{array}{c}\mathbf{F}_{2} \text { Population } \\
\text { Combination }\end{array}$ & $\begin{array}{c}\text { Total } \\
\text { Plants }\end{array}$ & $\begin{array}{c}\text { Fertile } \\
\text { Plants (F) }\end{array}$ & $\begin{array}{c}\text { Sterile } \\
\text { Plants (S) }\end{array}$ & F/S Ratio & $\chi^{2}$ & $\boldsymbol{P}$ & $\begin{array}{c}\text { Significant Test } \\
\boldsymbol{p}>\mathbf{0 . 0 5}\end{array}$ \\
\hline$m s 20 \times$ Chang7-2 & 1365 & 1065 & 300 & $3.55: 1$ & 3.47 & 0.06 & $\mathrm{~ns}$ \\
\hline
\end{tabular}

\subsection{Defective Anther Cuticle and Abortion Pollen Grain in ms20 Mutant}

To explore defects of $m s 20$ anther and pollen, scanning electron microscope (SEM) analysis was performed at maize anthesis stage. Consistent with the above morphological results, $m s 20$ anther was smaller and wilted (Figure 1B2) compared with that of WT (Figure 1B1). In addition, both the outer surface (Figure 1B4) and inner surface (Figure 1B6) of ms20 anther were glossy and smooth, and no typical Spaghetti-like anther cutin pattern (Figure 1B3) and Ubisch bodies (Figure 1B5) were observed in $m s 20$ anther. In WT, pollen grains with round shape (Figure 1A5,B7,C1) were generated for double fertilization, while only severely wizened pollen grains were retained and pasted on the inner wall of the anther locule (Figure 1A6,B8,C2).

\subsection{Isolation of $\mathrm{ZmMs} 20$}

The $\mathrm{F}_{2}$ population derived from the cross of $m s 20$ mutant $\times$ Chang7- 2 was used for gene mapping of the $m s 20$ locus. Primary mapping was performed by using a maize 6K SNP chip and two genomic DNA bulked pools from ten fertile and sterile $F_{2}$ individuals, respectively. Chromosome 1 was first selected as the candidate chromosome of the $m s 20$ locus, since more than $80-\mathrm{Mb}$ successive regions on its long arm have polymorphic ratios of $>40 \%$ (Figure 2A). Therefore, the $m s 20$ locus was primary-mapped to the long arm of chromosome 1 . Then, $96 \mathrm{~F}_{2}$ individuals were randomly selected and used for gene mapping to verify the above result based on the SNP chip. Consequently, the ms20 locus was located in a 4.8-cM region between markers bnlg2295 and P2-04 (Figure 2B, Table S1). By using a large population, including 540 sterile $\mathrm{F}_{2}$ individuals, $m s 20$ locus was further narrowed down to a 190-kb interval between markers P9-08 and P9-12 (Figure 2C). Seven candidate genes were predicted in this region according to the B73 reference genome (AGPv4) (Figure 2D), including Zm0001d029683, which is the reported GMS gene, IPE1 [30]. 


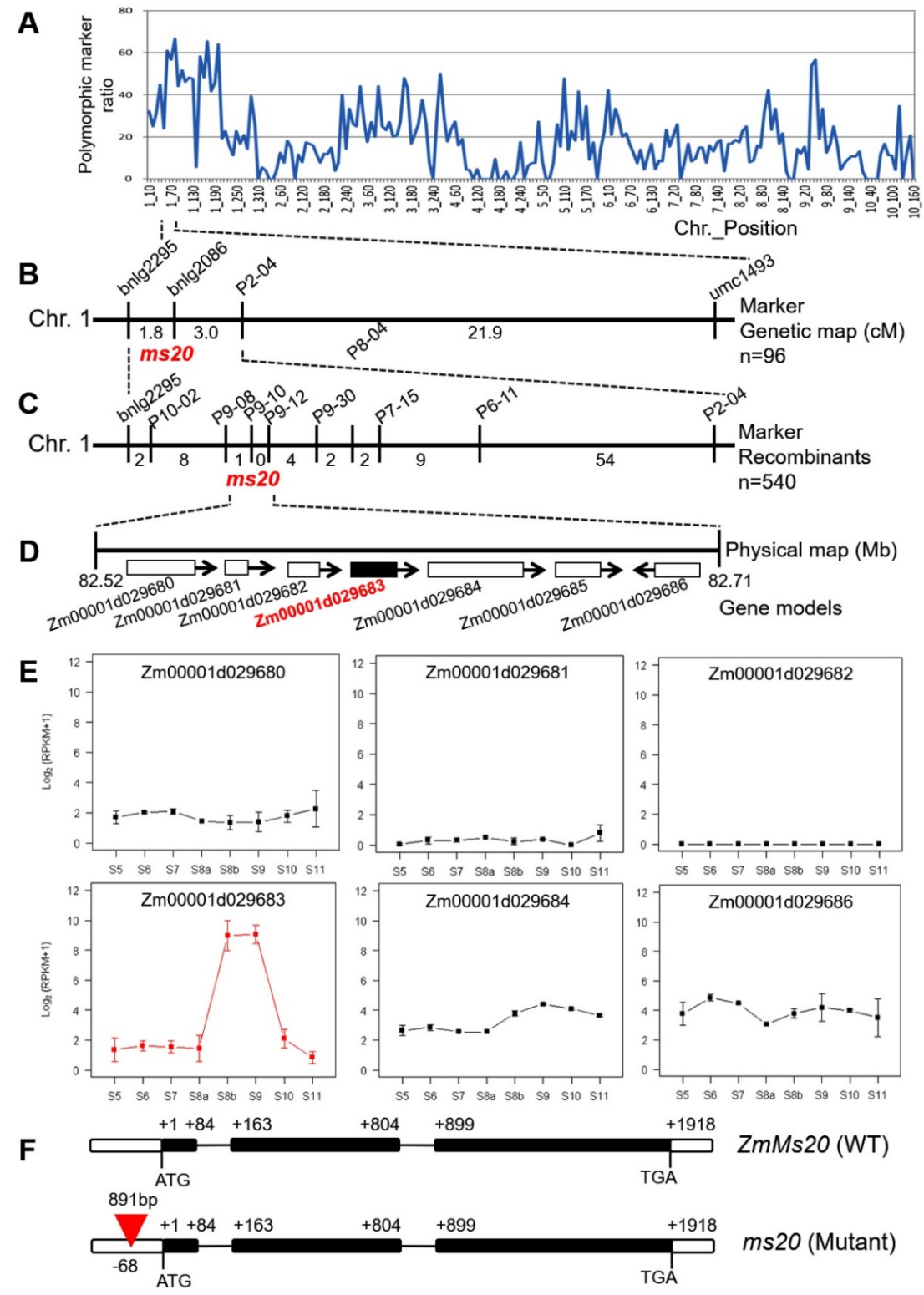

Figure 2. Map-based cloning of maize $m s 20$ gene, expression patterns of six candidate genes, and gene structure of $Z m M s 20$. (A) Polymorphic marker ratio of the sterile plant DNA pool (ms20/ms20) versus the fertile plant pool (ZmMs20/ZmMs20 and ZmMs20/ms20). (B) Primary mapping of $m s 20$ locus. The ms 20 locus was primarily mapped to the long arm of maize chromosome 1 between Simple Sequence Repeat (SSR) markers bnlg2295 and P2-04; n, the number of $F_{2}$ plants used for gene mapping, including 48 male-sterility and 48 male-fertility $\mathrm{F}_{2}$ individuals. (C) Fine mapping of $m s 20$ locus. The ms20 locus was narrowed down to a 190-kb interval between SSR markers P9-08 and P9-12; n, the number of male-sterility $\mathrm{F}_{2}$ individuals used for fine mapping. (D) The seven candidate gene models in the 190-kb interval. Among them, Zm00001d029683 was the IPE1 gene reported previously by Chen et al., 2017 [30]. (E) Expression patterns of six of the seven candidate genes in the 190-kb interval based on RNA sequencing data during 8 anther developmental stages (S5 to S11). Zm00001d029685 was annotated as a provisional gene and its expression was not detectable in the RNA sequencing data, whereas Zm00001d029683 was found to be high expression at both stages 8b and 9. (F) Gene structure of ZmMs20 and DNA sequence mutation of ms20. ZmMs20 consists of three exons and two introns. The +1 indicates the starting nucleotide site of translation, and the stop codon (TGA) is +1918 . Black boxes indicate exons, and intervening lines indicate introns. An 891-bp insertion at the $-68 \mathrm{bp}$ site of the $5^{\prime}$ UTR was found in the ms 20 mutant, and shown in a red triangle. 
Among the seven candidate genes, Zm00001d029685 was annotated as a provisional gene, and its expression was not detected in the following RNA-sequencing (RNA-seq) analysis. Expression patterns of the other six genes were analyzed based on RNA-seq data of maize anther during eight developmental stages (S5 to S11) (Figure 2E). Zm00001d029680, Zm00001d029684, and Zm00001d029686 keep a persistent expression at a relatively low level, and expression of Zm00001d029681 and Zm00001d029685 was almost undetectable in anther. Zm0001d029683 displayed high expression at S8b and S9 (Figure 2E). In addition, genomic DNA sequencing analysis of Zm0001d029683 between WT and $m s 20$ mutant revealed that an 891-bp fragment was inserted at the -68 bp site of the $5^{\prime}$ UTR in ms20 mutant and the coding region of Zm0001d029683 was identical to that of WT (Figure 2F), while no DNA sequence difference of the other expressed candidate genes was observed between WT and ms20 mutant. Therefore, all the results obtained above indicated that $Z m 0001 d 029683$ is the target gene and designated as $Z m M s 20$, which is a new allele of IPE1.

\subsection{Phylogenetic Evolution of ZmMs20}

ZmMs20 contains a 1743-bp open reading frame encoding 580 amino acids, and was predicted to encode a GMC oxidoreductase [30]. To illustrate the evolution relationship of ZmMs20, phylogenetic analysis was performed based on 19 orthologs in 11 plant species. The consequent neighbor-joining tree was clustered into two groups. The group I members were from monocots, and the group II members were from dicots (Figure 3A). Interestingly, only one ortholog was found in each of the selected monocot species, except wheat, which is hexaploidy and has 3 orthologs of ZmMs20. However, multiple orthologs were commonly presented in each of the selected dicot species except Arabidopsis (Figure 3A). This suggests that there may be different evolution pathways of $Z m M s 20$ and its orthologs between monocots and dicots.

GMC oxidoreductase is a small gene family in plants. 8, 7, and 6 members were found in Arabidopsis, rice, and maize, respectively. Multiple sequence alignment and phylogenetic analysis were performed by using the total $21 \mathrm{GMC}$ oxidoreductases in the three plants. Consequently, these GMC oxidoreductases were divided into 3 clades (Figure 3B). Clade I includes 7 members from rice and maize, and they are paired with each other for Os03t0118700. According to the previous reports, maize GMC family genes in clade I are specifically expressed in the tassel at the meiotic stage [32]. Arabidopsis At1g12570, an ortholog of ZmMs20, is the only member of clade II. In clade III, 13 members can be further divided into two subclades. Subclade III-1 includes 3 members from Arabidopsis, and subclade III-2 contains 10 members. Among the subclade III-2 members, MINI1/ONI3 (Os09t0363900) and ACE/HTH (At1g72970) were found to be involved in biosynthesis of long-chain fatty acids to prevent inappropriate fusions between neighboring floral organs [24,26].

Since all the reported GMC oxidoreductases in Arabidopsis and rice were involved in floral development, we analyzed expression patterns of the 6 GMC family genes in maize anther by using RNA-seq data during 8 developmental stages (Figure 3C, Table S2). Five of the 6 genes displayed high expression at certain anther stages, while the expression level of Zm00001d032284 was low, similar to its rice ortholog Os08t0401500, whose transcript was not detectable in rice [25]. Zm00001d002613, Zm0001d017598, and Zm00001d020238 displayed similar expression patterns to ZmMs20 with the expression peak at S9, while the expression of Zm00001d036701 appeared at S6, raised a plateau at S7 to $\mathrm{S} 8 \mathrm{~b}$, and disappeared at $\mathrm{S9}$ (Figure 3D). These results suggest that most of maize GMC oxidoreductases are perhaps involved in maize anther development. 
A

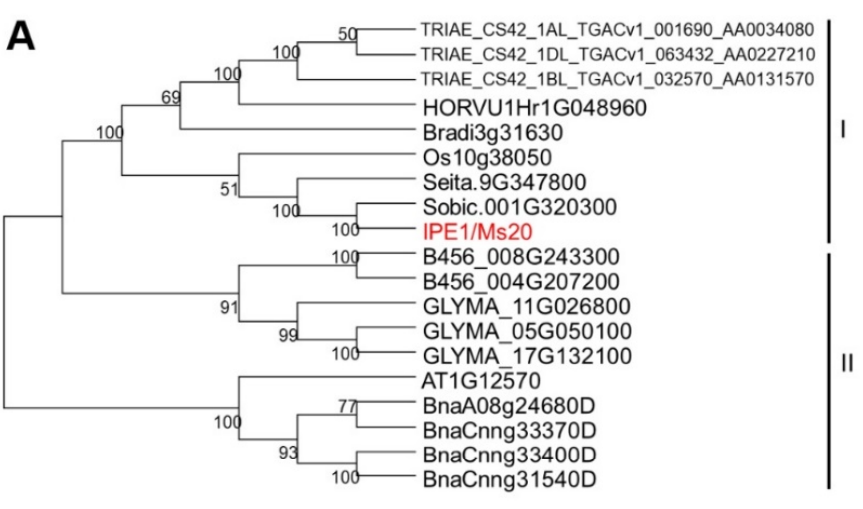

\begin{tabular}{ll|l|l|l|l|l|l|l|l} 
C & Stage & 5 & 6 & 7 & $8 \mathrm{a}$ & $8 \mathrm{~b}$ & 9 & 10 & 11
\end{tabular}
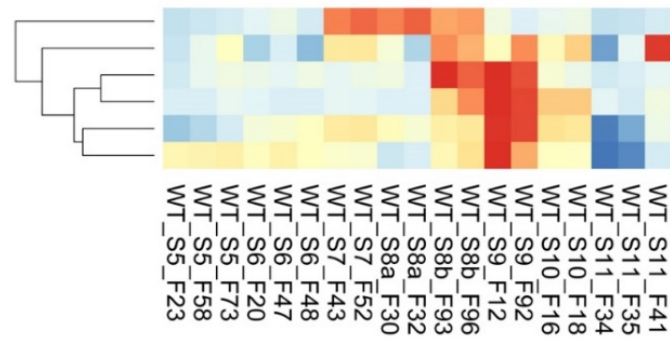

B

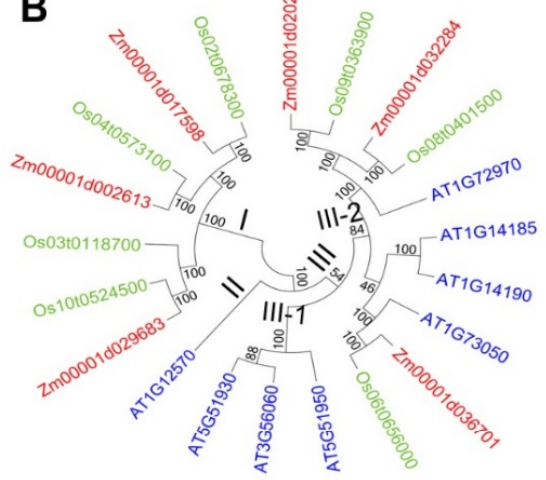

D14 Zm00001d036701, Zm00001d032284,

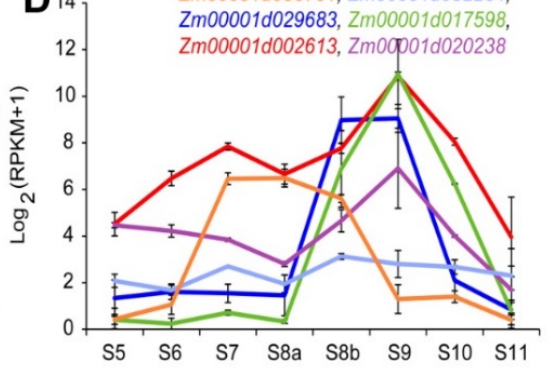

Figure 3. Phylogenetic analysis of ZmMs20 and its orthologs in different species and expression pattern analysis of ZmMs20 and its homologies in maize. (A) Phylogenetic analysis of ZmMs20 and its orthologs in different species. The analysis involved 19 amino acid sequences from Arabidopsis thaliana (At), Brassica napus (Bna, rape), Bradchypodium distachyon (Bradi, slender falsebrome), Hordeum vulgare (HORVU, barley), Gossypium raimondii (B, cotton), Glycine max (GLYMA, soybean), Oryza sativa (Os, rice), Setaria italica (Seita, millet), Sorghum bicolor (Sobic, sorghum), Triticum aestivum (TRIAE, wheat), and Zea mays (IPE1/Ms20, maize). The numbers on the branches represent the bootstrap values of the phylogenetic tree. The 19 orthologs can be divided into two groups. Group I comprises nine proteins from monocots, and group II includes ten proteins from dicots. (B) Phylogenetic tree of GMC family members in Arabidopsis, rice, and maize. A neighbor-joining tree showed the evolutionary relationships among GMC members in Arabidopsis (At), rice (Os), and maize ( $\mathrm{Zm})$. The GMC family members are named according to maize GDB or Phytozome accession number. The numbers under the branches represent the bootstrap values. (C) Hierachical clustering of six GMC family genes in maize based on RNA-seq data of anthers during 8 anther developmental stages (S5 to S11). (D) Expression patterns of $\mathrm{ZmMs} 20$ and its five homologies in maize.

In addition, microsynteny assay was performed by using flanking genes of ZmMs20 and its orthologs from six gramineous plants, including Brachypodium distachyon (slender falsebrome), Hordeum vulgare (barley), rice, Sorghum bicolor (sorghum), Setaria italica (millet), and maize (Table S3), and showed greatly significant synteny between $Z m M s 20$ and its orthologs among the six grasses (Figure 4). This result indicated that evolution of chromosomal regions harboring ZmMs20 and its orthologs is relatively conserved in these gramineous plants. 


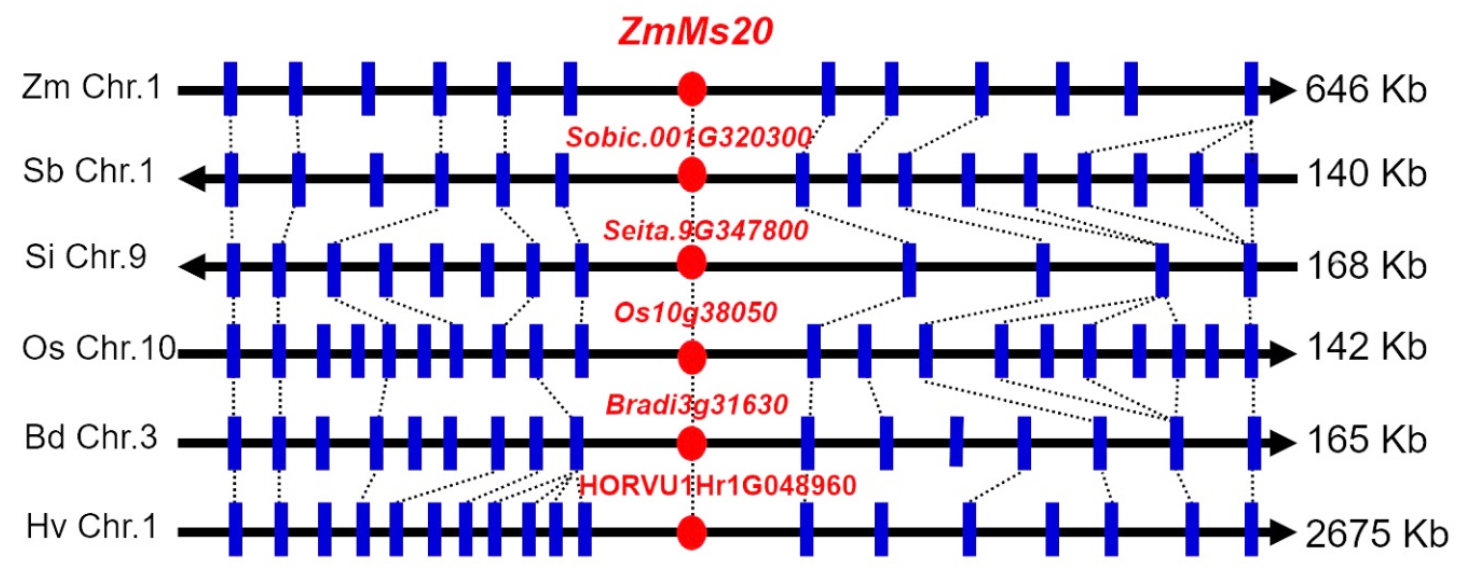

Figure 4. Microsynteny comparison of maize $\mathrm{ZmMs} 20$ locus with its orthologs in five other monocot species. Microsynteny comparison of maize ZmMs20 locus with its ortholog counterparts from Brachypodium distachyon (Bd), Hordeum vulgare (Hv), Oryza Sativa (Os), Sorghum bicolor (Sb), and Setaria italica (Si). The red solid circles indicate ZmMs20 and the orthologous genes, and the blue solid vertical lines indicate the genes flanking $Z m M s 20$ or the orthologous genes. The arrows indicate direction of the chromosomes (Chr.), and each gene in the flanking region was given a serial number (Refer to Table S2). The spanning region length between two loci was shown without equal proportion, and total length (kilobase, $\mathrm{kb}$ ) of the ortholog region was shown on the right.

\subsection{The Rigorous Stage-specificity Expression Patterns of GMS Genes in Maize}

Expression patterns of ZmMs20 were evaluated by using RNA-seq data of WT anthers from S5 to S11. The transcript level of ZmMs20 appeared at S8a and peaked at $8 \mathrm{~b}$ and S9 (Figure 5A,B4). To find out the sequential relationship of gene expression, we analyzed expression patterns of the 17 cloned GMS genes in maize and clustered these genes into four clades (Figure 5, Table S4). Clade I includes OCL4 and Ms22/MSCA1 [33,34], whose expression level peaked at S5 and became almost undetectable after $\mathrm{S} 6$ (Figure 5A,B1). Clade II contains MAC1, Ms32, and Ms33, whose expression started at $\mathrm{S} 5$ and peaked at $\mathrm{S} 6$, and then $M A C 1$ and $M s 32[35,36]$ were expressed in relatively low levels until S11, but expression of Ms33 [15] almost disappeared after S7 (Figure 5A,B2). Clade III is composed of Ms23, Ms9, Ms8, ZmMs30, and ms44 [17,37-40], which shared a similar expression plateau from S7 to S8b (Figure 5A,B3). Clade IV contains Ms45, Ms7, Ms20/IPE1, Ms26, APV1, IG1, and Ms6021 [7,10,13,20,30,31,41], whose expression peaked at $S 9$ and then decreased significantly (Figure 5A,B4). All the GMS genes displayed rigorous stage-specificity expression patterns during maize anther development, suggesting that different GMS genes may play important roles in regulating anther and pollen development and determining male fertility at their corresponding functioning stages in maize. 
A

$\begin{array}{lllllllll}\text { Stage } & 5 & 6 & 7 & 8 \mathrm{a} & 8 \mathrm{~b} & 9 & 10 & 11\end{array}$

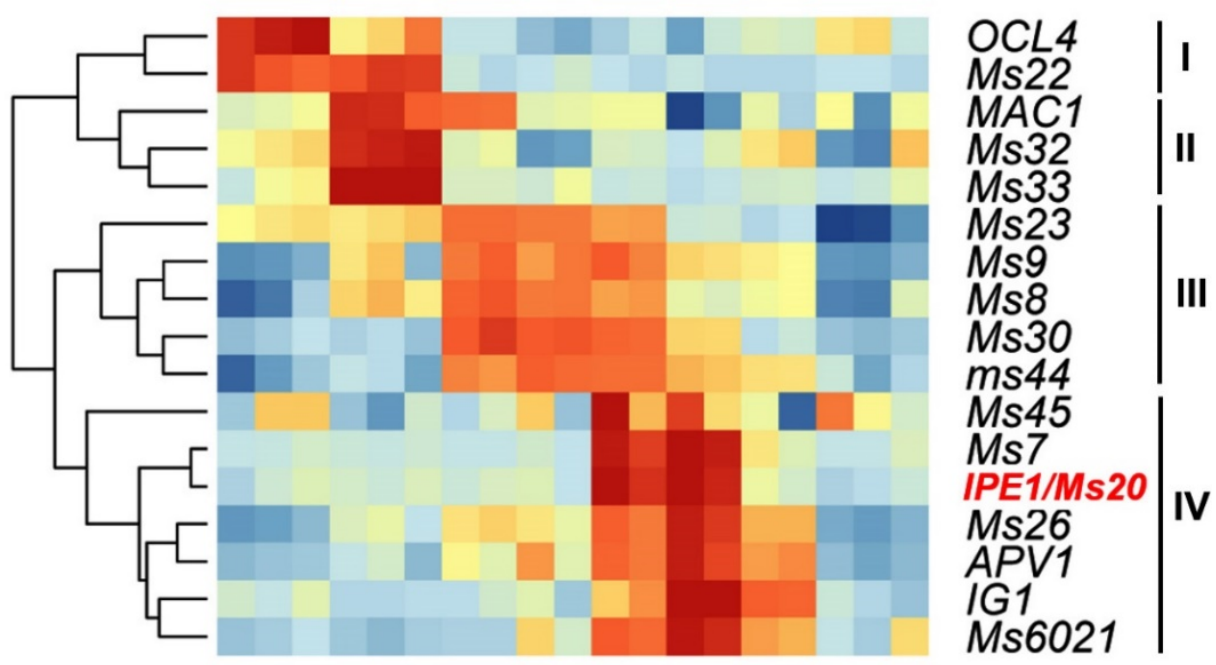

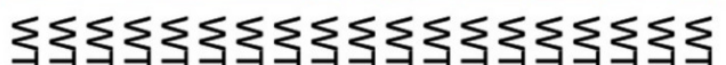

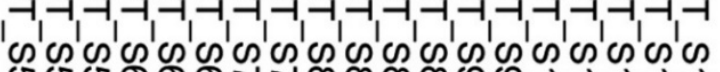

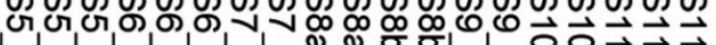

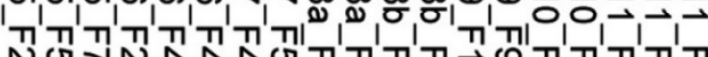

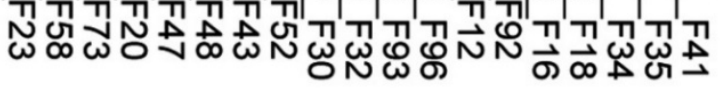

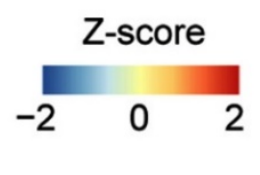

B
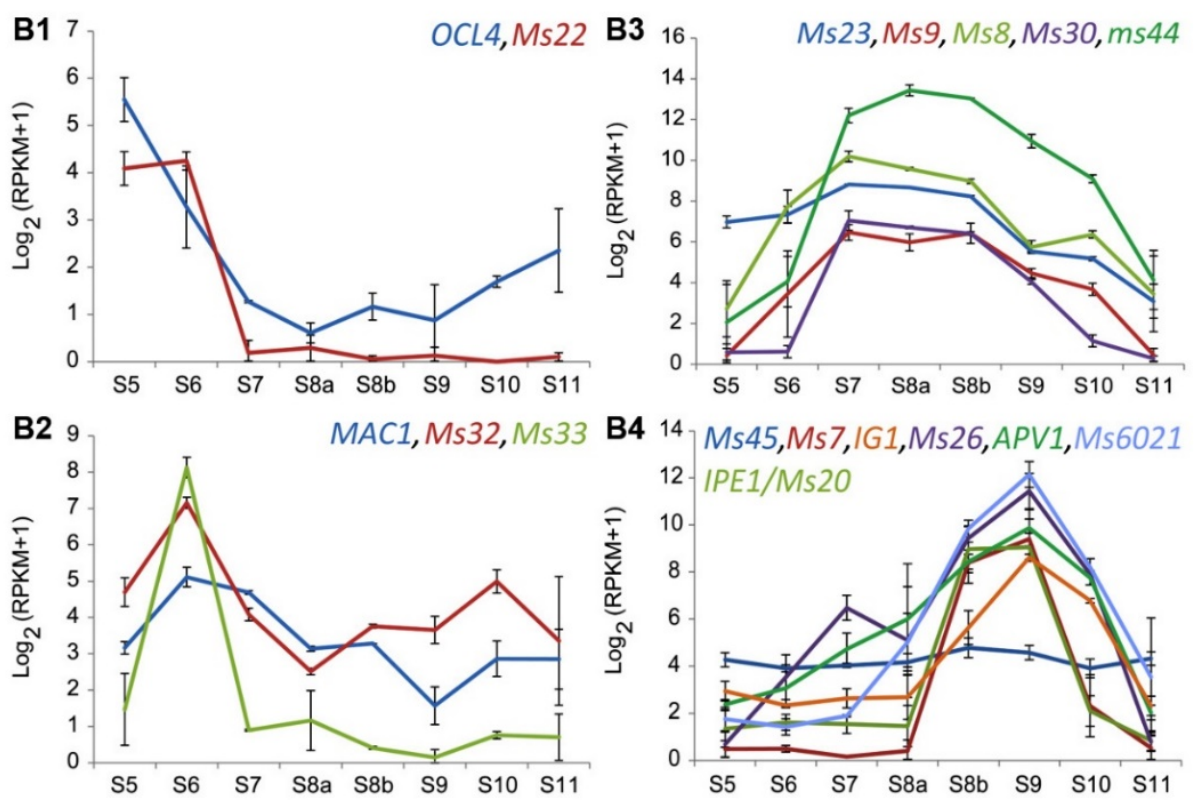

Figure 5. Expression patterns and classification of 17 GMS genes cloned in maize. (A) Hierachical clustering of the 17 cloned GMS genes based on RNA-seq data during eight anther developmental stages (S5 to S11). These GMS genes are clustered into four clades in chronological order of gene expression. Among them, ZmMs20/IPE1 is clustered into the clade IV. (B) Expression patterns of the GMS genes in clade I (B1), clade II (B2), clade III (B3), and clade IV (B4).

2.6. Two Co-segregating Functional Markers Developed for Creating ms20 Male-sterility Lines by MAS Strategy under Different Genetic Backgrounds

GMS lines have exhibited great value for hybrid breeding and seed production. Development of GMS lines can be accelerated by using co-segregating functional markers for MAS. Based on the information of 891-bp insertion in ms20 mutant, two makers, i.e., ms20-IN1 and ms20-IN2, were 
designed for MAS. The forward (1F) and reverse (1R) primers of ms20-IN1 were located upstream and downstream of 891-bp insertion, respectively (Figure 6A,B). The ms20-IN2 marker shared the forward primer $(1 \mathrm{~F})$ with ms20-IN1, while its reverse primer $(2 \mathrm{R})$ was located in the 891-bp insertion (Figure 6A,B). Based on this design, 204-bp and 1095-bp PCR products should be amplified by using a 1F/1R primer pair in homologous WT and ms20 mutant, respectively, and 487-bp PCR product should be amplified by using a $1 \mathrm{~F} / 2 \mathrm{R}$ primer pair in $m s 20$ mutant, but no PCR product could be detected in homologous WT (Figure 6B). The accuracy of two functional markers was further verified by using a PCR amplification test in three genotypic materials (ZmMs20/ZmMs20, ZmMs20/ms20, and $m s 20 / m s 20$ ) as templates (Figure $6 \mathrm{C}$ ). In addition, the co-segregating markers could differentiate genotypes of $\mathrm{ZmMs} 20$ and $m s 20$ in both $\mathrm{F}_{2}$ and $\mathrm{BC}_{1} \mathrm{~F}_{1}$ populations (Figure $6 \mathrm{D}, \mathrm{E}$ ). These results indicate that ms20-IN1 and ms20-IN2 markers can be used for MAS breeding to create $m s 20$ lines under different genetic backgrounds. To date, ms 20 mutation gene has been introduced into 353 different maize inbred lines by using these two function markers and the MAS strategy in our lab, and the obtained $353 \mathrm{~F}_{2}$ individuals with genotypes of $m s 20 / m s 20$ will be used to verify the male-sterility stability caused by ms20 mutation under different genetic backgrounds, based on the method as described in An et al., 2019 [17].

A

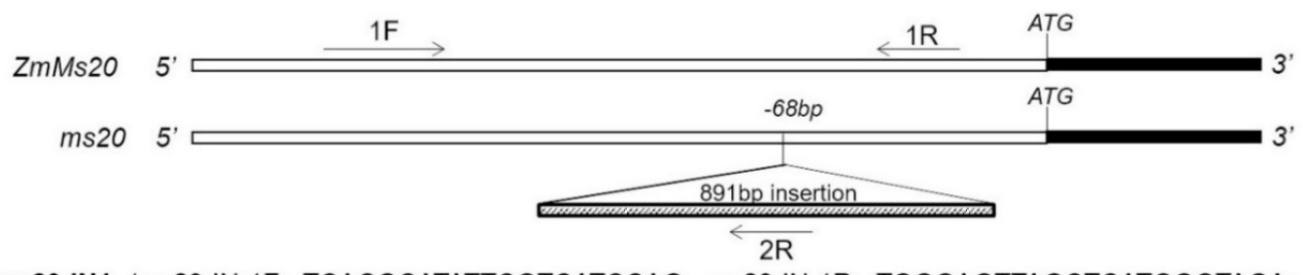

ms20-IN1 (ms20-IN-1F: TCACGCATATTCGTCATCCAG, ms20-IN-1R: TGGGAGTTAGCTCATGGGTAGA; ZmMs20: 204bp, $m s 20:$ 1095bp)

ms20-IN2 (ms20-IN-1F: TCACGCATATTCGTCATCCAG, ms20-IN-2R: AAGTGATAGGAAACAGGAAACCC; ZmMs20: no PCR product, $m s 20: 487 \mathrm{bp}$ )
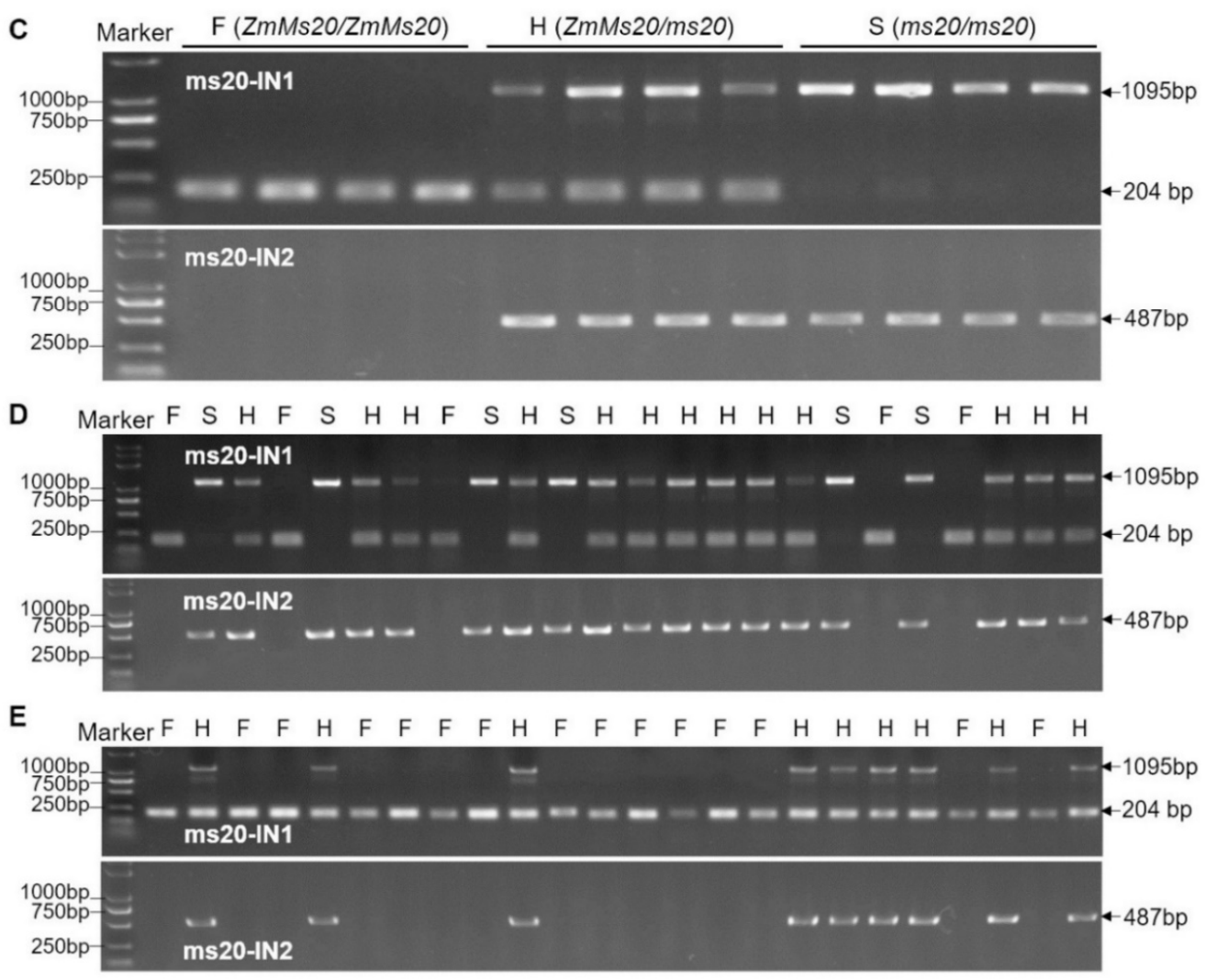

Figure 6. The functional markers of $\mathrm{ZmMs} 20 / m s 20$ and MAS of $m s 20$ mutant locus to introduce it into 
different genetic backgrounds. (A) A schematic representation of functional marker locations. The $1 F$ represents the common forward primer of ms20-IN1 and ms20-IN2 markers. The 1R represents the reverse primer of ms20-IN1 marker, and 2R represents the reverse primer of ms20-IN2 marker. (B) The primers' sequence and PCR production sizes of ms20-IN1 and ms20-IN2 markers. (C) Detection and verification of ms20-IN1 and ms20-IN2 markers. For ms20-IN1 marker, the larger bands with 1095-bp length indicate ms20 loci (sterility, S), the smaller bands with 204-bp length mean ZmMs20 loci (fertility, F), and $\mathrm{H}$ means heterozygous genotype of ZmMs20/ms20. For ms20-IN2 marker, the bands with 487-bp length indicate $m s 20$ loci (sterility, S), and the no PCR production means ZmMs20 loci (fertility, F). (D) Genotyping of $\mathrm{F}_{2}$ population derived from the cross and self-pollination of $m s 20$ mutant and a maize inbred line Zheng 58 using ms20-IN1 and ms20-IN2 markers. (E) Genotyping of $\mathrm{BC}_{1} \mathrm{~F}_{1}$ population derived from the cross and backcross of $m s 20 /$ Zheng 58//Zheng 58 using ms20-IN1 and ms20-IN2 markers.

\section{Discussion}

The $m s 20$, a complete male-sterility mutant, displayed smaller and wilted anther, a glossy anther outer surface, disappearance of Ubisch bodies, and aborted pollen grains (Figure 1). Its fertility restorer gene, ZmMs20, is a new allele of IPE1 [30] encoding a GMC oxidoreductase specifically expressed in maize anthers (Figure 2). Loss-function of IPE1 leads to similar defective phenotypes as ms20 mutant, for instance, absence of the typical Spaghetti-like anther cutin pattern, disappearance of Ubisch bodies, and arrested pollen exine accumulation [30]. Anther cuticle and pollen exine were considered as two important physical barriers protecting anther and pollen from various biotic and abiotic stresses [17], and their successful formation were found to be vital for anther and pollen development and male fertility.

The phylogenetic and microsynteny analyses revealed that ZmMs20 and its orthologs were conserved in gramineous plants (Figures 3 and 4). The identity of the amino acid was more than $88.8 \%$ among $\mathrm{ZmMs} 20$ and its orthologs. These results were consistent with the previous reports that the GMC domain was highly conserved and variations were primarily concentrated within the first 40 amino acids, which may contain a signal peptide [27]. OsNP1, an ortholog of ZmMs20, has been reported in rice [28,29]. Its mutant displays similar defective phenotypes to ms 20 mutant, such as undeveloped anther cuticle, disappearance of Ubisch bodies, and immature pollen exine. This implied conserved function between ZmMs20 and OsNP1. OsNP1 was predicted to take part in polymerization and assembly of sporopollenin and Ubisch bodies [29], which is essential for male reproduction processes. Therefore, we can conclude that orthologs of ZmMs20 in sorghum, millet, and wheat may also play similar roles in regulating anther and pollen development and male fertility. Knock-out of these orthologs possibly creates male-sterility genetic materials, which are useful for cross-breeding and hybrid seed production in crops.

ZmMs20 encodes a GMC oxidoreductase, a small gene family in plants. Phylogenetic analysis results indicated that GMC family genes might undergo different evolution pathways between monocots and dicots. Gene duplication had perhaps taken place in dicots to produce more GMC copies or members in dicots' genome (Figure 3). In addition, mutations of ZmMs20 orthologs result in different defects in Arabidopsis and rice, likely due to their functional differentiation. For instance, loss-function of $A C E / H T H$, the first GMC gene reported in Arabidopsis, leads to fusions of floral organs and genetic background-dependent male sterility due to the disruption of the membrane structure [24]. However, the early organ fusion of oni3/mini1 mutant is mainly observed in rice vegetative organs, and results in severe defects, such as seedling lethality [25,26]. Arabidopsis At1g12570, an ortholog of ZmMs20 and OsNP1, is the key regulator of cutin biosynthesis [42]. The T-DNA insertion mutant of At1g12570 (SALK-085330C) exhibits normal anther cuticle development and pollen grain extrusion as WT, even though a quantity of smaller pollen grains is observed in the mutant and the surface of some pollen grains with normal sizes is smooth [30]. The mutant phenotypes are greatly different from those of $m s 20$ (Figure 1) and $n p 1$ [29]. Taken together, it can be concluded that GMC-domain genes in dicots may retain partial functions for regulating anther and pollen development when compared to 
their corresponding orthologs in monocots, or the GMC genes are functionally redundant in dicots for gene duplication.

In maize, six GMC oxidoreductases were found according to the B73 reference genome (AGPv4). Apart for Zm00001d032284, whose ortholog (Os08t0401500) expression was undetectable in rice anther, all five other GMC genes displayed stage-specific expression patterns based on the RNA-seq analysis (Figure 3C,D). This indicates that the GMC family genes mainly contribute to anther development in maize, and thus systematic research on this gene family will provide clues to uncover how GMC family genes take part in the genetic regulation networks and bio-chemical pathways associated with anther development in maize.

All the maize GMS genes reported previously belong to either transcription factor or aliphatic enzymes. These GMS genes showed rigorous stage-specific expression patterns during anther development (Figure 5). Transcripts of ZmMs20 and the clade-IV genes encoding aliphatic enzymes were found to appear after initiation of meiosis, peak at S9, and then decline radically (Figure 5B4). Therefore, exploring regulators or interaction partners of ZmMs 20 could contribute to deeply understanding their roles in regulating anther development. In addition, Figure 5 clearly indicates that there are certain aliphatic enzyme genes controlling anther development at each stage from S5 to S9, since the expression peak of these genes appears at S5 to S9 separately. This implies that multiple aliphatic enzymes function in sequence on anther development. The aliphatic metabolisms in anther tapetum can produce and provide precursors for anther cuticle and pollen exine formation [43], which are essential for male fertility. However, the metabolism pathways are very sophisticated and the related bio-chemical mechanism is still unclear. Therefore, deciphering the in vivo substrates of these aliphatic enzymes and exploring the functional interaction relationship among these genes would greatly benefit our understanding of bio-chemical processes during anther development. ZmMs20/IPE1 was predicted to act downstream of ZmMs26, and could convert the $\omega$-hydroxy $\mathrm{C} 16 / \mathrm{C} 18$ fatty acids into $\mathrm{C} 16 / \mathrm{C} 18$ diacids. $\mathrm{C} 16: 0$ and $\mathrm{C} 18: 2$ diacids were significantly reduced in the ipe1 mutant anther [30], while C16 and C18 diacids only account for less than $1 \%$ of the total amount of cutin. How the trace of cutin component plays a vital role for anther development is still unknown. Therefore, more and deeper exploration needs to be performed in the future work to evaluate the exact substrates and catalytic activities of ZmMs20 and other aliphatic enzymes.

Finally, GMS genes and their corresponding mutants are valuable for hybrid breeding and seed production in crops. Most recently, the multi-control sterility (MCS) system has been developed, appraised, and updated by using ZmMs7, ZmMs33, and ZmMs30 and their male-sterility mutants in our laboratory $[16,17,20]$. This provides an opportunity to tactfully settle some matters and effectively utilize GMS genes for hybrid vigor utilization and hybrid seed production. Hence, the further exploration of new GMS genes is of great significance. For breeding application of ZmMs20 gene and ms20 mutant, two functional markers were developed to facilitate creating more ms 20 male-sterility lines under wide genetic backgrounds based on MAS strategy (Figure 6).

In summary, this work contributes to both mechanism deciphering of anther development and breeding application of the GMS gene and its mutant for creating more male-sterility lines, which can be used for hybrid vigor utilization and seed production in maize.

\section{Materials and Methods}

\subsection{Plant Materials, Growth Condition, and Phenotypic Characterization}

The $m s 20$ (No. 928C) mutant seeds were originally obtained from the Maize Genetics Operation Stock Center (http://maizecoop.cropsci.uiuc.edu). All the plant materials were grown in the experiments stations of University of Science and Technology Beijing (USTB) in Beijing and Hainan Province. Tassels were photographed with an EOS 7000 digital camera (Canon, Tokyo, Japan). Anthers and pollen grains stained with 1\% I2-KI solution were photographed with a 
SZX2-ILLB stereomicroscope (Olympus, Tokyo, Japan) and BX-53F microscope (Olympus, Tokyo, Japan), respectively.

\subsection{SEM Analysis}

For SEM observation, the fresh anthers of both WT and ms20 mutant were immersed in FAA solution (Coolabor, Beijing, China) overnight for fixation. The anthers were then dehydrated in an ethanol series (50\%, 75\%, 95\%, and 100\%) for 15 min each step. After critical-point drying, the samples were coated with palladium gold in an ion sputter (JFC, Osaka, Japan) and observed under a SEM (HITACHI S, Tokyo, Japan).

\subsection{Genetic Analysis and Map-Based Cloning of ZmMs20}

$\mathrm{F}_{2}$ segregating population was derived from the cross of $m s 20 \times$ Chang7-2. Genetic analysis was performed by calculating the segregating ratio of fertile to sterile $\mathrm{F}_{2}$ individuals. Genomic DNA was extracted from maize leaves using a modified method [44].

To map ms20 locus on chromosomes, ten $\mathrm{F}_{2}$ individuals of each phenotype (male-fertility and sterility plants) were randomly selected for constructing a fertility bulked DNA pool (ZmMs20) and male-sterility bulked DNA pool (ms20). A SNP polymorphism analysis was performed by using maize 6K SNP chip (Compass Biotechnology, Beijing, China) assay, and genotyping was performed according to the manufacturer's recommendations using the Illumina iScan System (Illumina, San Diego, CA, USA). The polymorphic ratios between WT and ms 20 bulked pools in the 10-cM region along the chromosome was calculated, and the successive region with a high polymorphic ratio was considered as the candidate flanked region of $\mathrm{ZmMs} 20$.

For gene mapping of ms 20 locus, $96 \mathrm{~F}_{2}$ individuals were screened with SSR markers in the candidate-linked region. Genotyping data of these markers and $\mathrm{F}_{2}$ individual phenotypes were analyzed by using the Joinmap 4.0 program. Subsequently, fine mapping of $m s 20$ locus was carried out by using $540 \mathrm{~F}_{2}$ individuals with male-sterility phenotype and the developed SSR markers based on the B73 reference genome (AGPv4). Finally, the ms20 locus was mapped to a 190-kb region on chromosome 1. Within this region, Zm00001d029683 was considered as the target gene of ZmMs20. It was previously reported as IPE1 [30].

\subsection{Sequence Alignment and Phylogenetic Analysis}

Multiple sequence alignment of maize ZmMs20 with its corresponding orthologs in other plants were performed in the CLUSTALX program [45]. The phylogenic tree was reconstructed in the Molecular Evolutionary Genetics Analysis (MEGA6) program using the maximum likelihood method [46]. Support values were estimated by 1000 times of bootstrap replicates.

\subsection{Expression Analysis and Clustering of the Maize Cloned GMS Genes Using RNA-seq}

For RNA-seq analysis, total RNAs of maize WT anthers from S5 to S11 were extracted using RNeasy Plant Mini Kit (Qiagen, Dusseldorf, Germany). Each sample was used to create libraries that were deep-sequenced using the Illumina HiSeq 2500 System (Illumina, San Diego, CA, USA) to generate 150-bp, paired-end reads. Reads were trimmed based on quality scores $(p \geq 15)$ and adapter sequences were removed. Reads were mapped to the maize reference genome (AGPv4) using TopHat2.0 with default parameters [47]. Aligned reads were counted with Rsubread and then quantified and normalized with edgeR [48,49]. Normalized expression is shown in RPKM (read per kb per million mapped reads). Pheatmap program was used to generate heat maps of expression levels of the 17 cloned GMS genes and further perform clustering and classification. 


\subsection{Microsynteny Analysis of ZmMs20}

For microsynteny analysis, neighboring genes of $Z m M s 20$ were identified in the maize reference genome (AGPv4), and then the flanking genes of ZmMs20 orthologs in sorghum, millet, rice, barley, and slender falsebrome were downloaded from Phytozome V12 (https:/ / phytozome.jgi.doe.gov/pz/ portal.html) (Table S3). Microsynteny of ZmMs20 was determined by multiple sequence alignments of the flanking genes surrounding $Z m M s 20$ or its orthologs among the six grasses.

\subsection{Development of the Co-segregating Markers of ZmMs20/ms20 for MAS Breeding}

Based on the sequence differences of ZmMs20 between WT and ms 20 mutant, two co- segregating markers, ms20-IN1 and ms20-IN2, were designed and tested by PCR amplifications with gel electrophoresis. The two markers could be used for MAS breeding of the $m s 20$ locus to introduce it into maize of different genetic backgrounds. All the PCR primers described above are listed in Table S1.

Supplementary Materials: Supplementary materials can be found at http:/ /www.mdpi.com/1422-0067/20/6/ $1411 /$ s1.

Author Contributions: Conceptualization, X.W.; data curation and Investigation, Y.W., D.L., Y.T., S.Z., and J.B.; formal analysis, Y.W., Z.L., and D.L.; funding acquisition, X.W., project administration, J.L.; resources, S.W.; software, Z.L. and Z.D.; supervision, X.A.; writing-original draft, Y.T., D.L., and X.W.; writing-review and editing, X.W.

Funding: This research was funded by the National Transgenic Major Program of China (2018ZX0800922B and 2018ZX0801006B), the National Key Research and Development Program of China (2017YFD0102001, 2018YFD0100806, and 2017YFD0101201), the National Natural Science Foundation of China (31771875 and 31871702), the Fundamental Research Funds for the Central Universities of China (06500060, FRF-TP-18-013A1, FRF-TP-18-014A1), the "Ten Thousand Plan"-National High Level Talents Special Support Plan (For Xiangyuan Wan), and the Beijing Talents Foundation From Organization department of Beijing Municipal committee of CPC (For Xiangyuan Wan, No. 2016000021223TD04).

Acknowledgments: We thank Yanbao Tian (Institute of Genetics and Developmental Biology, Chinese Academy of Sciences) for help in SEM sample preparation.

Conflicts of Interest: The authors declare no conflict of interest.

\section{Abbreviations}

$\begin{array}{ll}\text { CMS } & \text { Cytoplasmic Male Sterility } \\ \text { GMC } & \text { Glucose Methanol Choline } \\ \text { GMS } & \text { Genic Male Sterility } \\ \text { MAS } & \text { Marker Assisted Selection } \\ \text { MCS, } & \text { Multi-Control Sterility } \\ \text { RNA-seq } & \text { RNA-sequencing } \\ \text { SEM } & \text { Scanning Electron Microscope } \\ \text { SSR } & \text { Simple Sequence Repeat } \\ \text { WT } & \text { Wild Type }\end{array}$

\section{References}

1. Wan, X.Y.; Wu, S.W.; Li, Z.W.; Dong, Z.Y.; An, X.L.; Ma, M.; Tian, Y.H.; Li, J.P. Maize genic male-sterility genes and their applications in hybrid breeding: Progress and Perspectives. Mol. Plant 2019, 12, 321-342. [CrossRef] [PubMed]

2. Yeats, T.H.; Rose, J.K. The formation and function of plant cuticles. Plant Physiol. 2013, 163, 5-20. [CrossRef] [PubMed]

3. Heredia, A. Biophysical and biochemical characteristics of cutin, a plant barrier biopolymer. Biochim. Biophys. Acta 2003, 1620, 1-7. [CrossRef]

4. Walton, T.I.; Harwood, J.L.; Bowyer, J.R. Methods in Plant Biochemistry: Lipids, Membranes and Aspects of Photobiology; Academic Press: San Diego, CA, USA, 1990; Volume 4, pp. 105-158. 
5. Chen, W.; Yu, X.H.; Zhang, K.; Shi, J.; De Oliveira, S.; Schreiber, L.; Shanklin, J.; Zhang, D.B. Male Sterile2 encodes a plastid-localized fatty acyl carrier protein reductase required for pollen exine development in Arabidopsis. Plant Physiol. 2011, 157, 842-853. [CrossRef]

6. Shi, J.; Tan, H.; Yu, X.H.; Liu, Y.; Liang, W.; Ranathunge, K.; Franke, R.B.; Schreiber, L.; Wang, Y.; Kai, G.; et al. Defective pollen wall is required for anther and microspore development in rice and encodes a fatty acyl carrier protein reductase. Plant Cell 2011, 23, 2225-2246. [CrossRef]

7. Tian, Y.; Xiao, S.; Liu, J.; Somaratne, Y.; Zhang, H.; Wang, M.; Zhang, H.R.; Zhao, L.; Chen, H.B. MALE STERILE6021 (MS6021) is required for the development of anther cuticle and pollen exine in maize. Sci. Rep. 2017, 7, 16736. [CrossRef]

8. Morant, M.; Jorgensen, K.; Schaller, H.; Pinot, F.; Moller, B.L.; Werck-Reichhart, D.; Bak, S. CYP703 is an ancient cytochrome $\mathrm{P} 450$ in land plants catalyzing in-chain hydroxylation of lauric acid to provide building blocks for sporopollenin synthesis in pollen. Plant Cell 2007, 19, 1473-1487. [CrossRef] [PubMed]

9. Yang, X.; Wu, D.; Shi, J.; He, Y.; Pinot, F.; Grausem, B.; Yin, C.; Zhu, L.; Chen, M.; Luo, Z. Rice CYP703A3, a cytochrome $\mathrm{P} 450$ hydroxylase, is essential for development of anther cuticle and pollen exine. J. Integr. Plant Biol. 2014, 56, 979-994. [CrossRef] [PubMed]

10. Somaratne, Y.; Tian, Y.; Zhang, H.; Wang, M.; Huo, Y.; Cao, F.; Zhao, L.; Chen, H.B. ABNORMAL POLLEN VACUOLATION1 (APV1) is required for male fertility by contributing to anther cuticle and pollen exine formation in maize. Plant J. 2017, 90, 96-110. [CrossRef] [PubMed]

11. Dobritsa, A.A.; Shrestha, J.; Morant, M.; Pinot, F.; Matsuno, M.; Swanson, R.; Moller, B.L.; Preuss, D. CYP704B1 is a long-chain fatty acid omega-hydroxylase essential for sporopollenin synthesis in pollen of Arabidopsis. Plant Physiol. 2009, 151, 574-589. [CrossRef]

12. Li, H.; Pinot, F.; Sauveplane, V.; Werck-Reichhart, D.; Diehl, P.; Schreiber, L.; Franke, R.; Zhang, P.; Chen, L.; Gao, Y. Cytochrome P450 family member CYP704B2 catalyzes the $\omega$-hydroxylation of fatty acids and is required for anther cutin biosynthesis and pollen exine formation in rice. Plant Cell 2010, 22, 173-190. [CrossRef]

13. Djukanovic, V.; Smith, J.; Lowe, K.; Yang, M.; Gao, H.; Jones, S.; Nicholson, M.G.; West, A.; Lape, J.; Bidney, D. Male-sterile maize plants produced by targeted mutagenesis of the cytochrome P450-like gene (MS26) using a re-designed I-CreI homing endonuclease. Plant J. 2013, 76, 888-899. [CrossRef]

14. Men, X.; Shi, J.; Liang, W.; Zhang, Q.; Lian, G.; Quan, S.; Zhu, L.; Luo, Z.; Chen, M.; Zhang, D. Glycerol-3-Phosphate Acyltransferase 3 (OsGPAT3) is required for anther development and male fertility in rice. J. Exp. Bot. 2017, 68, 513-526. [CrossRef]

15. Xie, K.; Wu, S.; Li, Z.; Zhou, Y.; Zhang, D.; Dong, Z.; An, X.; Zhu, T.; Zhang, S.; Liu, S.; et al. Map-based cloning and characterization of Zea mays male sterility33 (ZmMs33) gene, encoding a glycerol-3-phosphate acyltransferase. Theor. Appl. Genet. 2018, 131, 1363-1378. [CrossRef]

16. Zhu, T.T.; Wu, S.W.; Zhang, D.F.; Li, Z.W.; Xie, K.; An, X.L.; Ma, M.; Hou, Q.C.; Dong, Z.Y.; Tian, Y.H.; et al. Genome-wide analysis of maize GPAT gene family and cytological characterization and breeding application of ZmMs33/ZmGPAT6 gene. Theor. Appl. Genet. 2019, TAAG-D-18-00637R1. (Accepted).

17. An, X.L.; Dong, Z.Y.; Tian, Y.H.; Xie, K.; Wu, S.W.; Zhu, T.T.; Zhang, D.F.; Zhou, Y.; Niu, C.F.; Ma, B.; et al. $\mathrm{ZmMs} 30$ encoding a novel GDSL lipase is essential for male fertility and valuable for hybrid breeding in maize. Mol. Plant 2019, 12, 343-359. [CrossRef]

18. Yang, C.; Vizcay-Barrena, G.; Conner, K.; Wilson, Z.A. MALE STERILITY1 is required for tapetal development and pollen wall biosynthesis. Plant Cell 2007, 19, 3530-3548. [CrossRef]

19. Li, H.; Yuan, Z.; Vizcay-Barrena, G.; Yang, C.; Liang, W.; Zong, J.; Wilson, Z.A.; Zhang, D. PERSISTENT TAPETAL CELL1 encodes a PHD-finger protein that is required for tapetal cell death and pollen development in rice. Plant Physiol. 2011, 156, 615-630. [CrossRef]

20. Zhang, D.; Wu, S.; An, X.; Xie, K.; Dong, Z.; Zhou, Y.; Xu, L.; Fang, W.; Liu, S.; Liu, S.; et al. Construction of a multicontrol sterility system for a maize male-sterile line and hybrid seed production based on the ZmMs7 gene encoding a PHD-finger transcription factor. Plant Biotechnol. J. 2018, 16, 459-471. [CrossRef]

21. Cavener, D.R. GMC oxidoreductases. A newly defined family of homologous proteins with diverse catalytic activities. J. Mol. Biol. 1992, 223, 811-814. [CrossRef]

22. Wongnate, T.; Chaiyen, $\mathrm{P}$. The substrate oxidation mechanism of pyranose 2-oxidase and other related enzymes in the glucose-methanol-choline superfamily. FEBS J. 2013, 280, 3009-3027. [CrossRef] [PubMed] 
23. Sorigue, D.; Legeret, B.; Cuine, S.; Blangy, S.; Moulin, S.; Billon, E.; Richaud, P.; Brugiere, S.; Coute, Y.; Nurizzo, D. An algal photoenzyme converts fatty acids to hydrocarbons. Science 2017, 357, 903-907. [CrossRef] [PubMed]

24. Kurdyukov, S.; Faust, A.; Trenkamp, S.; Bar, S.; Franke, R.; Efremova, N.; Tietjen, K.; Schreiber, L.; Saedler, H.; Yephremov, A. Genetic and biochemical evidence for involvement of HOTHEAD in the biosynthesis of long-chain $\alpha$ - $\omega$-dicarboxylic fatty acids and formation of extracellular matrix. Planta 2006, 224, 315-329. [CrossRef] [PubMed]

25. Akiba, T.; Hibara, K.; Kimura, F.; Tsuda, K.; Shibata, K.; Ishibashi, M.; Moriya, C.; Nakagawa, K.; Kurata, N.; Itoh, J. Organ fusion and defective shoot development in oni3 mutants of rice. Plant Cell Physiol. 2014, 55, 42-51. [CrossRef] [PubMed]

26. Fang, Y.; Hu, J.; Xu, J.; Yu, H.; Shi, Z.; Xiong, G.; Zhu, L.; Zeng, D.; Zhang, G.; Gao, Z. Identification and characterization of Mini1, a gene regulating rice shoot development. J. Integr. Plant Biol. 2015, 57, 151-161. [CrossRef] [PubMed]

27. Xu, Y.; Liu, S.; Liu, Y.; Ling, S.; Chen, C.; Yao, J. HOTHEAD-Like HTH1 is Involved in anther cutin biosynthesis and is required for pollen fertility in rice. Plant Cell Physiol. 2017, 58, 1238-1248. [CrossRef] [PubMed]

28. Chang, Z.; Chen, Z.; Wang, N.; Xie, G.; Lu, J.; Yan, W.; Zhou, J.; Tang, X.; Deng, X.W. Construction of a male sterility system for hybrid rice breeding and seed production using a nuclear male sterility gene. Proc. Natl. Acad. Sci. USA 2016, 113, 14145-14150. [CrossRef] [PubMed]

29. Liu, Z.; Lin, S.; Shi, J.; Yu, J.; Zhu, L.; Yang, X.; Zhang, D.; Liang, W. Rice No Pollen 1 (NP1) is required for anther cuticle formation and pollen exine patterning. Plant J. 2017, 91, 263-277. [CrossRef]

30. Chen, X.; Zhang, H.; Sun, H.; Luo, H.; Zhao, L.; Dong, Z.; Yan, S.; Zhao, C.; Liu, R.; Xu, C. IRREGULAR POLLEN EXINE1 is a novel factor in anther cuticle and pollen exine formation. Plant Physiol. 2017, 173, 307-325. [CrossRef]

31. Evans, M.M. The indeterminate gametophyte1 gene of maize encodes a LOB domain protein required for embryo sac and leaf development. Plant Cell 2007, 19, 46-62. [CrossRef]

32. Winter, D.; Vinegar, B.; Nahal, H.; Ammar, R.; Wilson, G.V.; Provart, N.J. An “Electronic Fluorescent Pictograph" browser for exploring and analyzing large-scale biological data sets. PLoS ONE 2007, 2, e718. [CrossRef]

33. Vernoud, V.; Laigle, G.; Rozier, F.; Meeley, R.B.; Perez, P.; Rogowsky, P.M. The HD-ZIP IV transcription factor OCL4 is necessary for trichome patterning and anther development in maize. Plant J. 2009, 59, 883-894. [CrossRef] [PubMed]

34. Albertsen, M.; Fox, T.; Trimnell, M.; Wu, Y.; Lowe, L.; Li, B.; Faller, M. Msca1 Nucleotide Sequences Impacting Plant Male Fertility and Method of Using Same. U.S. Patent US20090038027A1, 5 Feburary 2009.

35. Wang, C.J.; Nan, G.L.; Kelliher, T.; Timofejeva, L.; Vernoud, V.; Golubovskaya, I.N.; Harper, L.; Egger, R.; Walbot, V.; Cande, W.Z. Maize multiple archesporial cells 1 (mac1), an ortholog of rice TDL1A, modulates cell proliferation and identity in early anther development. Development 2012, 139, 2594-2603. [CrossRef] [PubMed]

36. Moon, J.; Skibbe, D.; Timofejeva, L.; Wang, C.J.; Kelliher, T.; Kremling, K.; Walbot, V.; Cande, W.Z. Regulation of cell divisions and differentiation by MALE STERILITY32 is required for anther development in maize. Plant J. 2013, 76, 592-602. [CrossRef] [PubMed]

37. Nan, G.L.; Zhai, J.; Arikit, S.; Morrow, D.; Fernandes, J.; Mai, L.; Nguyen, N.; Meyers, B.C.; Walbot, V. MS23, a master basic helix-loop-helix factor, regulates the specification and development of the tapetum in maize. Development 2017, 144, 163-172. [CrossRef] [PubMed]

38. Albertsen, M.; Fox, T.; Leonard, A.; Li, B.; Loveland, B.; Trimnell, M. Cloning and Use of the ms9 Gene from Maize. U.S. Patent US20160024520A1, 8 January 2016.

39. Wang, D.X.; Skibbe, D.S.; Walbot, V. Maize Male sterile 8 (Ms8), a putative $\beta$-1,3galactosyltransferase, modulates cell division, expansion, and differentiation during early maize anther development. Plant Reprod. 2013, 26, 329-338. [CrossRef] [PubMed]

40. Fox, T.; DeBruin, J.; Haug, C.K.; Trimnell, M.; Clapp, J.; Leonard, A.; Li, B.; Scolaro, E.; Collinson, S.; Glassman, K. A single point mutation in Ms44 results in dominant male sterility and improves nitrogen use efficiency in maize. Plant Biotechnol. J. 2017, 15, 942-952. [CrossRef] 
41. Cigan, A.M.; Unger, E.; Xu, R.J.; Kendall, T.; Fox, T.W. Phenotypic complementation of ms45 maize requires tapetal expression of MS45. Sex. Plant Reprod. 2001, 14, 135-142. [CrossRef]

42. Kannangara, R.; Branigan, C.; Liu, Y.; Penfield, T.; Rao, V.; Mouille, G.; Hofte, H.; Pauly, M.; Riechmann, J.L.; Broun, P. The transcription factor WIN1/SHN1 regulates cutin biosynthesis in Arabidopsis thaliana. Plant Cell 2007, 19, 1278-1294. [CrossRef]

43. Ariizumi, T.; Toriyama, K. Genetic regulation of sporopollenin synthesis and pollen exine development. Annu. Rev. Plant Biol. 2011, 62, 437-460. [CrossRef]

44. Zhou, X.Y.; Li, L.Z.; Xiang, J.H.; Gao, G.F.; Xu, F.X.; Liu, A.L.; Zhang, X.W.; Peng, Y.; Chen, X.B.; Wan, X.Y. OsGL1-3 is involved in cuticular wax biosynthesis and tolerance to water deficit in rice. PLOS ONE 2015, 10, e116676. [CrossRef]

45. Higgins, D.G.; Thompson, J.D.; Gibson, T.J. Using CLUSTAL for multiple sequence alignments. Methods Enzymol. 1996, 266, 383-402. [CrossRef]

46. Tamura, K.; Stecher, G.; Peterson, D.; Filipski, A.; Kumar, S. MEGA6: Molecular Evolutionary Genetics Analysis version 6.0. Mol. Biol. Evol. 2013, 30, 2725-2729. [CrossRef]

47. Trapnell, C.; Pachter, L.; Salzberg, S.L. TopHat: Discovering splice junctions with RNA-Seq. Bioinformatics 2009, 25, 1105-1111. [CrossRef]

48. Robinson, M.D.; McCarthy, D.J.; Smyth, G.K. edgeR: A Bioconductor package for differential expression analysis of digital gene expression data. Bioinformatics 2010, 26, 139-140. [CrossRef]

49. Liao, Y.; Smyth, G.K.; Shi, W. Feature Counts: An efficient general purpose program for assigning sequence reads to genomic features. Bioinformatics 2014, 30, 923-930. [CrossRef]

(C) 2019 by the authors. Licensee MDPI, Basel, Switzerland. This article is an open access article distributed under the terms and conditions of the Creative Commons Attribution (CC BY) license (http:/ / creativecommons.org/licenses/by/4.0/). 